\section{Menopause and hormone replacement therapy in the 21st century}

\author{
Felice L Gersh, ${ }^{1}$ Carl J Lavie (1) ${ }^{2}$
}

There continues to be controversy regarding the use of menopausal therapy for women, with considerable evidence supporting the benefits of oestrogen for women, ${ }^{1}$ but with recent guidelines from the American Heart Association stating that hormone replacement therapy (HRT) and selective oestrogen-receptor modulators should not be used for the primary or secondary prevention of cardiovascular $(\mathrm{CV})$ disease (CVD). ${ }^{2}$ Nevertheless, there is substantial data to support that premature menopause is associated with increased risk of coronary heart disease (CHD) and CVD events. ${ }^{3}$ The reason for this, however, remains uncertain, including the adverse effects of earlier loss of oestrogen on CHD risk factors or loss of other beneficial effects of oestrogen on the CV system.

In their Heart paper, O'Keeffe and colleagues ${ }^{4}$ use data from the large United Kingdom Medical Research Counsel National Survey of Health and Development to examine the association between age at cessation of menstrual period and trajectory of CHD risk factors from ages 53-69 years in over 2500 women, where approximately 1670 had data available on blood pressure and body composition and approximately 1500 had data on lipids and glucose metabolism. These results did not demonstrate a clear impact of cessation of period on conventional CHD and CVD risk factors, therefore, suggesting that the reason for the increased CHD and CVD risk in these women with early menopause likely comes from other factors.

Whether or not a woman enters menopause at age 50 or 53 years may not have a great deal of differential impact on her overall CV status by the time she reaches age 69. There is, however, no doubt that with the arrival of menopause, the progression of the insidious diseases of ageing, and of CV dysfunction, accelerate. ${ }^{3}$ Indeed, CVD events remain the number one cause of death of American women.

${ }^{1}$ Department of Medicine, Division of Integrative Medicine, University of Arizona College of Medicine, Tucson, Arizona, USA

${ }^{2}$ Cardiology, John Ochsner Heart and Vascular Institute, New Orleans, Louisiana, USA

Correspondence to Dr Carl J Lavie, Cardiology, John Ochsner Heart and Vascular Institute, New Orleans, LA 70121, USA; clavie@ochsner.org dinucleotide phosphate.
Menopause signifies far more than the cessation of monthly cycles, it marks the loss of ovarian oestrogen production, a great force disrupting cardio-metabolic homeostasis. Oestrogen modulates vascular tone and protects the vascular endothelium via anti-inflammatory, antioxidant and anti-proliferative pathways, and its loss contributes to reduced nitric oxide production, oxidative stress and vascular dysfunction (figure 1). ${ }^{56}$

Despite the numerous published articles on the myriad CV protective roles played by oestrogen in the premenopausal woman, it remains entrenched in the medical management of menopausal women that HRT, if prescribed at all, should remain a short-term solution, using the lowest dose possible to minimise night sweats, and only be considered for systemic use to address night sweats and hot flashes, rather than to maintain optimal $\mathrm{CV}$ function. The unintended legacy of the Women's Health Initiative (WHI), which tested the wrong 'hormones' (not human-identical and given orally), on the wrong subjects (too old), must now finally be ended.

The large body of scientific data that shows the harms of ovarian senescence and the $\mathrm{CV}$ benefits derived from hormones must now override the negative data derived from the WHI. It should not be a surprise that the combination of an oral conjugated oestrogen derived from pregnant horse urine, blended with a progesterone endocrine disruptor, medroxyprogesterone acetate, would not yield the comparable beneficial effects of endogenous female human-identical hormones. Our current knowledge of oestrogen metabolism and of oestrogen receptors provides a clear rationale for the failure of the WHI to demonstrate definitive therapeutic benefits, as the agents used in the study were alien to a human female body and were delivered in a non-physiologic manner. Only the use of hormone combinations identical to that found in a female body-transdermal human-identical estradiol combined with human-identical progesterone would be expected to yield clear CV benefits. In addition to not using human-identical oestradiol and progesterone, the use of oral oestrogen derived from horse urine increased blood clotting and inflammatory markers and was metabolised to the less beneficial estrone and differing metabolites compared with transdermal oestradiol use.

A great body of data exists concerning the many roles played by estradiol in maintaining cardio-metabolic homeostasis. Estradiol, the principle oestrogen produced by the ovaries, supports all aspects of the CV system: the arterial endothelium, the myocardium, mitochondrial oxidative phosphorylation and other

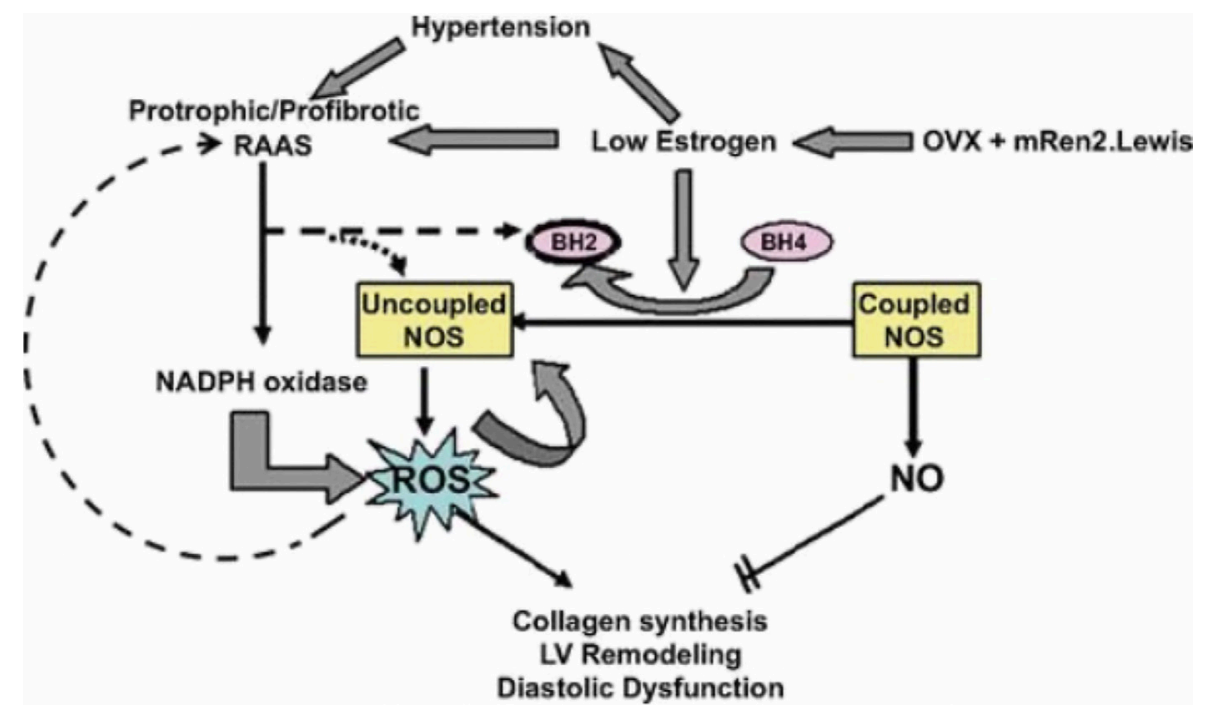

Figure 1 Diagrammatic representation of the pathways by which oestrogen deficiency induces hypertension, promotes the development of free radicals and leads to collagen synthesis, left ventricular remodelling and diastolic dysfunction. ${ }^{6}$ RAAS, renin-angiotensin-aldosterone system; NO, nitric oxide; NOS, nitric oxide synthase, ROS, reactive oxygen species; LV,left ventricular; $\mathrm{OVX}$, ovariectomy; $\mathrm{BH} 2$, dihydrobiopterin; $\mathrm{BH} 4$, tetrahydrobiopterin; $\mathrm{NADPH}$, nicotinamide adenine 
mitochondrial functions, nitric oxide production in arteries and a great many other essential functions. Oestrogen, as estradiol, modulates mitochondrial ATP synthesis in the heart and decreases stressinduced mitochondrial reactive oxygen species production. ${ }^{7}$ Oestrogen prevents apoptosis and necrosis of cardiac and endothelial cells and reduces pathologic cardiac hypertrophy. ${ }^{1}$ Oestrogen protects the premenopausal heart from hypertension and ventricular remodelling, and there is a sharp increase in the prevalence of left ventricular diastolic dysfunction after menopause. ${ }^{6}$ Males and females manifest very differently with heart failure (HF), 'Published reports show that estrogen deficiency influences both early diastolic relaxation ... and the late diastolic compliance associated with cardiac hypertrophy and fibrosis. Because of the high risk of diastolic dysfunctional in HF with preserved ejection fraction [HFpEF] in post-menopausal women and the positive effects of estrogen on preserving cardiac function, further clinical studies are needed to clarify the role of endogenous estrogen or hormone replacement in mitigating the onset and progression of $\mathrm{HFpEF}$ in women.' ${ }^{8}$ The loss of ovarian function that evolves through the perimenopausal and menopausal years elevates a woman's risk of CVD events in ways quite distinct from male hearts of similar age. Women's hearts are more susceptible to fibrosis and oxidative stress (figure 2). ${ }^{9}$

Though there remains a limited number of quality studies evaluating the most efficacious application of HRT, the current state of research is enough to justify the use of human-identical HRT with most women as they develop ovarian senescence. Numerous conditions linked to both ageing and estrogen deficiency, such as joint damage from osteoarthritis, vision loss due to cataracts, tooth loss due to periodontal disease and fractures from osteoporosis, are readily treated by the medical community, so why should the recognised harms of menopause to the CV system not be proactively treated with HRT?

The time has arrived to shelve the WHI and its limited applicability to the lives of menopausal women. Human-identical transdermal estradiol and micronised progesterone should be offered to most menopausal women, particularly at the menopause initiation. Menopause has a powerful negative impact on the $\mathrm{CV}$ health of women. They deserve to be offered HRT, using hormones identical to those produced by human ovaries, and the hormones should be prescribed in the most physiological manner possible. More research is needed, but it is time now to

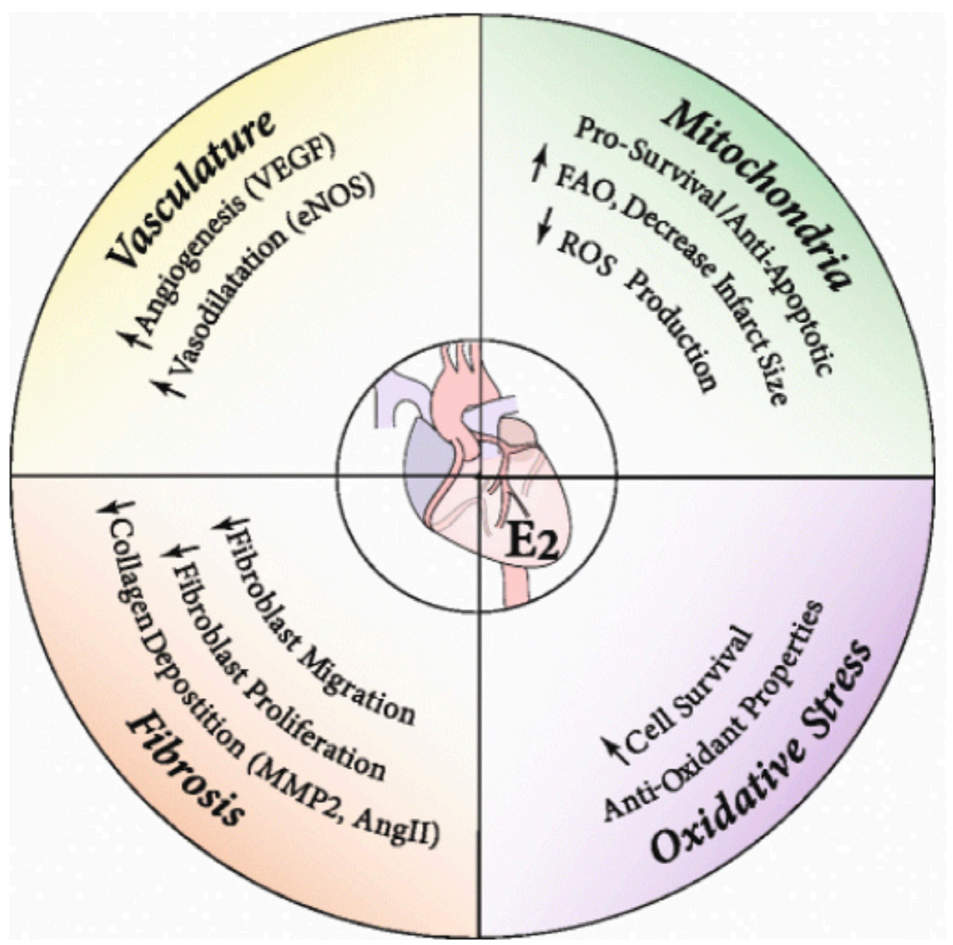

Figure 2 Illustration of the myriad ways that estradiol impacts cardiac health: supporting mitochondrial function and vascular health; reducing cardiac remodelling and oxidative stress. ${ }^{9}$ VEGF,vascular endothelial growth factor;eNOS, endothelial nitric oxide synthase; Angll angiotensin II, MMP2, matrix metalloproteinase 2; ROS, reactive oxygen species; FAO, fatty acid oxidation. acknowledge the impact of menopauseof ovarian senescence-on the CV health of women and treat them with physiological doses of human-identical hormones, prior to the development of serious CVD sequela.

Besides the limitations that the authors mentioned, particularly too few clinical events to adequately analyse, they were also not able to provide details regarding the HRT administration or potential use of cardioprotective medications that may have helped risk factors, and the age cut-points for follow-up were fairly arbitrary. Nevertheless, these data certainly suggest CHD and CVD risk factors do not explain the increased CVD risk from early menopause, and likely these adverse effects come from the loss of other beneficial effects of oestrogen, as we discussed above. More importantly, however, we believe that many women would benefit from more effective, safe and physiologic HRT as discussed above, and this is especially needed for women with earlier menopause.

Contributors All statements below apply to both authors: substantial contributions to the conception or design of the work, or the acquisition, analysis or interpretation of data.Drafting the work or revising it critically for important intellectual content. Final approval of the version published. Agreement to be accountable for all aspects of the work in ensuring that questions related to the accuracy or integrity of any part of the work are appropriately investigated and resolved.

Funding The authors have not declared a specific grant for this research from any funding agency in the public, commercial or not-for-profit sectors.

Competing interests None declared.

Patient consent for publication Not required.

Provenance and peer review Commissioned; internally peer reviewed.

(c) Author(s) (or their employer(s)) 2020. No commercial re-use. See rights and permissions. Published by BMJ.

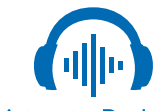

Listen to Podcast heart.bmj.com

D Check for updates

To cite Gersh FL, Lavie CJ. Heart 2020;106:479-481.

Published Online First 25 February 2020

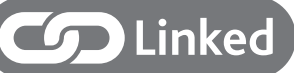

- http://dx.doi.org/10.1136/heartjnl-2019-315754

Heart 2020;106:479-481.

doi:10.1136/heartjnl-2019-315898

ORCID iD

Carl J Lavie http://orcid.org/0000-0003-3906-1911 


\section{REFERENCES}

1 Knowlton AA, Lee AR. Estrogen and the cardiovascular system. Pharmacol Ther 2012;135:54-70.

2 Mosca L, Benjamin EJ, Berra K, et al. Effectivenessbased guidelines for the prevention of cardiovascular disease in women--2011 update: a guideline from the american heart association. Circulation 2011:123:1243-62.

3 Muka T, Oliver-Williams C, Kunutsor S, et al. Association of age at onset of menopause and time since onset of menopause with cardiovascular outcomes, intermediate vascular traits, and all-cause mortality: a systematic review and meta-analysis. JAMA Cardiol 2016;1:767-76.

4 O'Keeffe LM, Kuh D, Fraser A, et al. Age at period cessation and trajectories of cardiovascular risk factors across mid and later life: a prospective cohort study. Heart 2020;106:499-505.

5 Chakrabarti S, Lekontseva O, Davidge ST. Estrogen is a modulator of vascular inflammation. IUBMB Life 2008;60:376-82.

6 Zhao Z, Wang $H$, Jessup JA, et al. Role of estrogen in diastolic dysfunction. Am J Physiol Heart Circ Physiol 2014;306:H628-40.
7 Rattanasopa C, Phungphong S, Wattanapermpool J, et al. Significant role of estrogen in maintaining cardiac mitochondrial functions. J Steroid Biochem Mol Biol 2015;147:1-9.

8 Beale AL, Nanayakkara S, Segan L, et al. Sex Differences in Heart Failure With Preserved Ejection Fraction Pathophysiology: A Detailed Invasive Hemodynamic and Echocardiographic Analysis. JACC Heart Fail 2019;7:239-49.

9 lorga A, Cunningham CM, Moazeni S, et al. The protective role of estrogen and estrogen receptors in cardiovascular disease and the controversial use of estrogen therapy. Biol Sex Differ 2017;8:33. 